\title{
Lightning Impulse Discharge Behaviour in Tẫ-Water Gap
}

\author{
Daisuke Okano \\ Liberal Arts Education Center, Tokai University \\ 9-1-1 TorokuHigashiku, Kumamoto, 862-8652 Japan
}

\begin{abstract}
When the soil invaded by water as the grounding medium in green power generation sites near river basins, on the coast, is dominantly characterized in terms of its resistivity, the characteristics of the voltage and current waveforms on transient discharges such as the soil can be experimentally investigated using the liquid gap, reproducing the resistivity of the soil. The transient discharge characteristics is available information on the surge withstand rating of surge protective devices used in the green power generation sites. Experimental results on the behaviour of lightning impulse discharges in a tap-water gap are reported; these results show that the resistivity of tap water is nearly equal to those of a river and cultivated and marshy land. Further, (1) the discharge voltage and current waveforms indicate the periodic attenuated waveforms for one period, and the voltage waveforms indicate amplitude distortions; (2) the discharge impedance obtained using the fitting curves to the discharge waveforms ranges from 1 to 2.5 times that of the tap-water resistance, i.e., $80 \Omega_{2 .}$ and from 18 , to 3.2 times that for positive and negative rod discharges.
\end{abstract}

Index Terms: lightning, impulse discharge, grounding resistivity, tap̃ water

\section{Introduction}

Most green energy facilities such as photovoltaic or wind power generation systems have been constructed near river and lake basins [1], [2], on the coast [3], or offshore [4] to secure both the air flow required annually and the wide building sites for high power output due to system integration [5] or to avert low-frequency noise pollution originating from the wind turbine operation [6]. This means that power generation buildings are likely to be affected by lightning surges via space, ground, and river or sea invasion routes [7], [8]. From the viewpoint of internal lightning protection [9], [10], it is important to evaluate the efficiency of surge protective devices (SPDs) [11], [12], as applied on the electrical power systems of the power generation systems. The internal protection measures against lightning attacks need to be verified in each discharge medium, given that the voltage and current waveforms and their impedance vary individually with the discharge media [13].

The soil in the grounding medium has been electrically specified on the basis of its resistivity and permittivity [14]. These are called the "soil parameters," and they affect the lightning response of grounding electrodes. The soil impedance [15] decreases with an increase in the water content ratio to the soil volume when the soil is invaded by water, and the resistance component can be experimentally approximated to the soil impedance [14] at high frequencies in lightning strokes. Then, the liquid medium gap in discharges is available for obtaining a desired resistivity by adjusting the salt concentration in pure water. The tap water in Japan shows a resistivity of $50 \Omega \mathrm{m}$ and is a candidate for a gap medium of lightning discharges when the resistivity of the grounding soil near a river and a lake of less than 100 $\Omega \mathrm{m}$ is to be reproduced [15].

The impedance can be obtained by the proposed method [16] using instantaneous values in discharge voltage and current waveforms. When a voltage waveform $(V)$ precedes a current one $(I)$ in any medium, the equivalent circuit can be approximately represented as a simple series circuit consisting of resistance and inductance. Then the basic equation is generally

Received: September $15^{\text {th }}, 2012$. Accepted: May $27^{\text {th }}, 2013$ 
given by $R I_{\mathrm{k}}+L \mathrm{~d} I_{\mathrm{k}} / \mathrm{dt}=V_{\mathrm{k}}$ fork $=1,2$, considering the simultaneous voltage equation in the series circuit with the series impedance $Z(=R+j X X X \equiv 2 \pi L / T, T:$ waveform period $)$ consisting of the resistance $(R)$ and inductance $(L)$. The simultaneous equation is also consisted of $R I_{1}+L$ $\mathrm{d} I_{1} / \mathrm{dt}=V_{1}$ and $R I_{2}+L \mathrm{~d} I_{2} / \mathrm{dt}=V_{2}$ for the time interval $\left(\Delta t_{\mathrm{s}}\right), \Delta t_{\mathrm{s}}=\left|t_{2}-t_{1}\right|$.

Our standard lightning impulse generator (SLIG, $1.2 / 50 \mu \mathrm{s}$ in waveforms) is classified as Marx Circuit with triple capacitor stage. The SLIG is a series circuit consisting of passive circuit elements: a capacitor, inductor, and resistor. It usually outputs a lightning voltage waveform with either positive or negative polarity. However, the SLIG also outputs a bipolar voltage waveform by satisfying the oscillation condition in a transient circuit when its output resistance is connected in parallel to a small resistance. Therefore, the oscillating waveforms of the voltage and current during the discharge may contain useful information for determining the discharge impedance. To determine the discharge impedance in the liquid gap, the impulse discharges were performed under oscillating conditions. In this study, to accumulate basic data for utilizing SPD, we examine the time dependence of the discharge waveform and impedance during an impulse discharge using a standard lightning impulse generator in a tap-water gap.

\section{Discharge Experiments}

The equivalent circuit for the SLIG used in the experiment is shown in Figure 1. It is a series circuit with passive circuit elements $C_{\mathrm{IG}}, r_{\mathrm{IG}}, L_{\mathrm{IG}}$, and $R_{\mathrm{IG}}$. $C_{\mathrm{IG}}$ represents the total capacitance, $66.7 \mathrm{nF}$, which is supplied by three $200 \mathrm{nF}$ capacitors connected serially. The total series resistance $r_{\mathrm{IG}}$ is $30 \Omega$. $L_{\mathrm{IG}}$ represents the inductance, which is $360 \mu \mathrm{H}$, and $R_{\mathrm{IG}}$ represents the output resistance of $992 \Omega$. To determine the discharge impedance in tap-water, we performed discharge experiments under the oscillating discharge condition through the SLIG. When $R_{\mathrm{IG}}$ is connected to the discharge impedance $\left(Z_{\mathrm{w}}\right)$ in parallel, the oscillation condition is fulfilled as $r_{\mathrm{IG}}+R_{\mathrm{IG}}^{\prime}<2\left(L_{\mathrm{IG}} / C_{\mathrm{IG}}\right)^{0.5}$ for $R_{\mathrm{IG}}^{\prime}=R_{\mathrm{IG}} Z_{\mathrm{w}} /\left(R_{\mathrm{IG}}+Z_{\mathrm{w}}\right) \sim Z_{\mathrm{w}}<<R_{\mathrm{IG}}$, where $Z_{\mathrm{w}}$ is assumed to be as small as the value for a lead wire.

The electrode set was consisted of the $\operatorname{rod}(\Phi 3.0 \mathrm{~mm} \times 50 \mathrm{~mm})$ and plate $(250 \mathrm{~mm} \times 250$ $\mathrm{mm}$ ) electrodes which were made of stainless steel (SUS304). The liquid gap between the rod and plate electrodes is also consisted of tap-water as shown in Figure $2(\mathrm{a})$. The liquid gap $\left(d_{\mathrm{w}}\right)$ filling with tap-water with a resistivity of $50 \Omega \mathrm{m}$ was $d_{\mathrm{w}}=100 \mathrm{~mm}$, where its static resistivity was determined using a conductivity meter (sensION ${ }^{\mathrm{TM}}$ 5,Hach Co. Ltd.) [17].

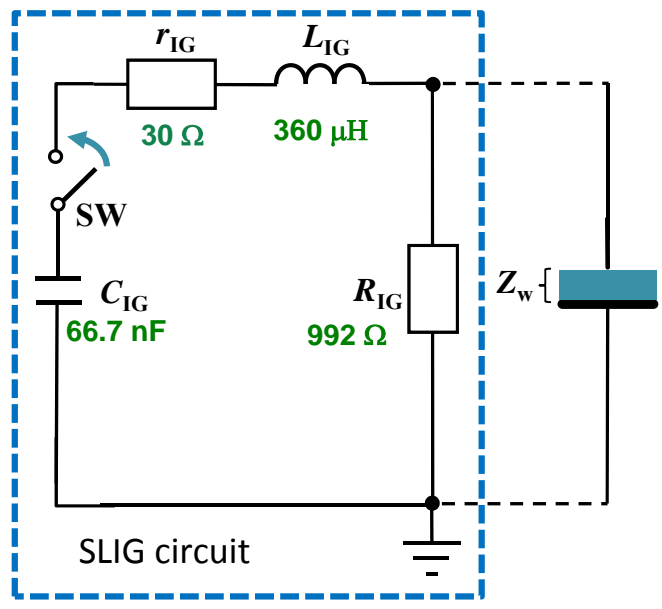

Figure 1. Schematic diagram of standard lightning impulse generator (SLIG) circuit, where $Z_{\mathrm{w}}$ denotes discharge impedance 
A set of voltage and current values was measured by a digital storage oscilloscope (DSO) as shown in Figure2 (b). The discharge voltage was measured by a high-voltage probe (HVP) using a resistor-divider with an attenuation ratio of 1/5000 and a response time of less than $1 \mathrm{~ns}$. The discharge current was measured by a high response current probe (HRCP) using a semiconductor sensor with an attenuation ratio of $1 / 100$ and a response time of 5 ns. The discharge emission photographs on the liquid were taken by a Polaroid camera (PC) located over the supporter in Figure 2 (b).

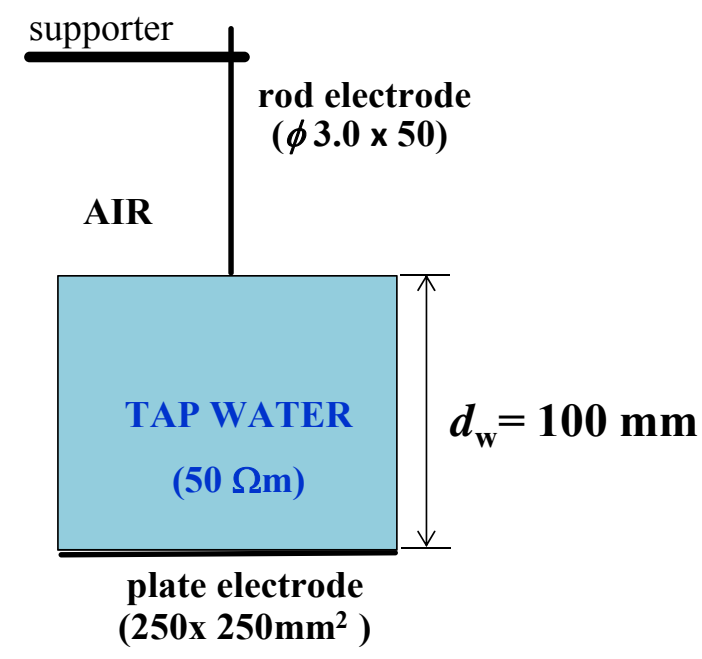

(a) tap-water gap

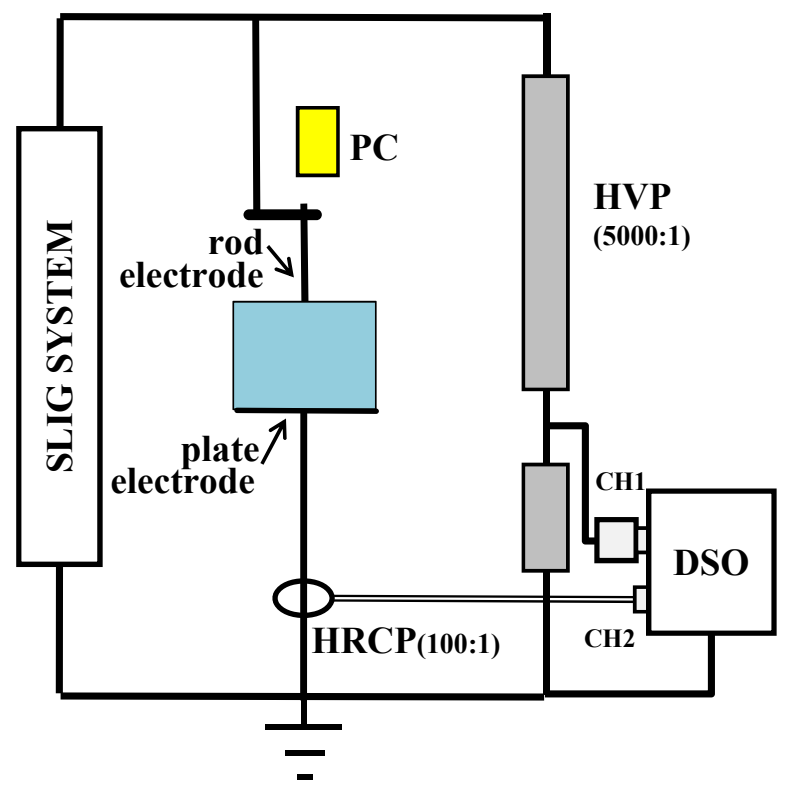

(b) impulse discharge circuit

Figure 2. Schematic diagram of (a) tapwwater gap and (b) impulse discharge circuitin experimental setup 


\section{Experimental Results and Discussion}

The emission photographs on the tap -water are shown in Figures 3 (a) and (b) for positive and negative rod discharges, respectively, where each shadow was caused by the supporter. The emission area for the positive discharge on the liquid was rather smaller than the negative case, because the experiment was done at different threshold voltages corresponding to the $50 \%$ flashover voltage $\left(V_{50}\right)$ as shown in Figures 4 (a) and (b). It was found that the positive discharge emission indicated many delicate branches compared to the negative case as well as those on dielectric solids.

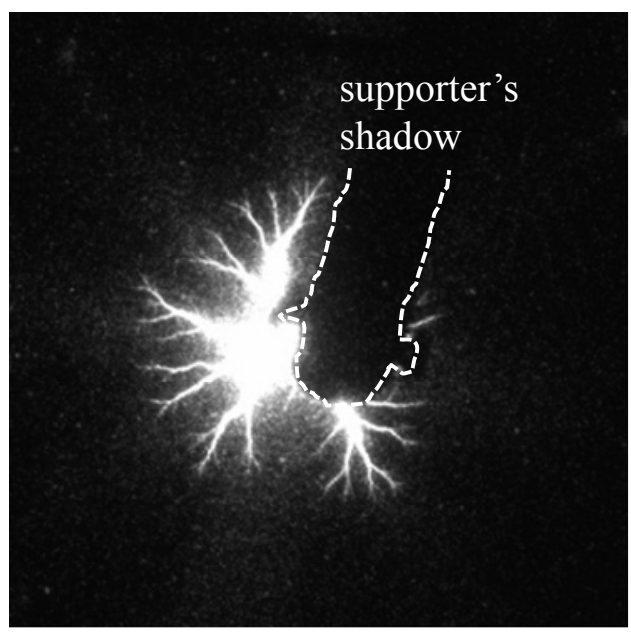

(a). positive rod

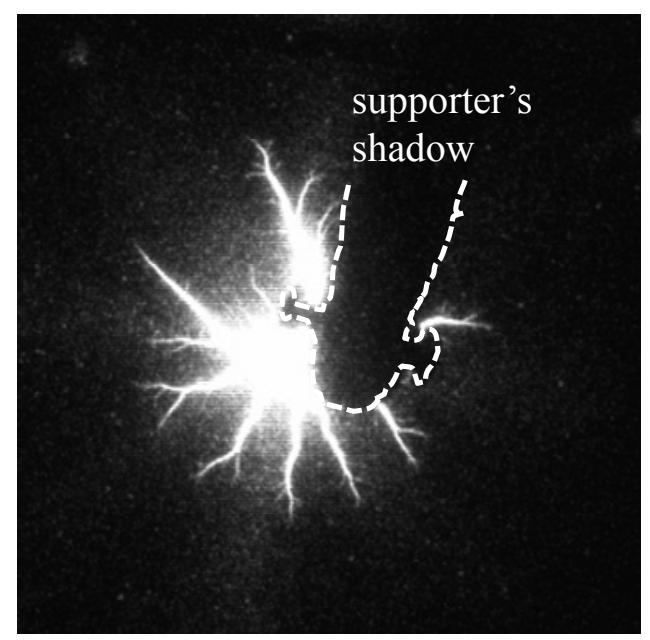

(b). negative rod

Figure 3. Photographs for (a) positive and (b) negative rod dischargeson tap-water

The discharge property of $V_{50}$ and time lag $\left(t_{\mathrm{d}}\right)$ in the tap-water ("TWATER" denoted in Figure 4) gap was compared to those in air and air-tap-water gaps as shown in Figures4 (a) and (b). It was found that the $t_{\mathrm{d}}$ was nearly equal to each other in all gaps while the $V_{50}$ in the tap-water gap indicated a minimum value. 


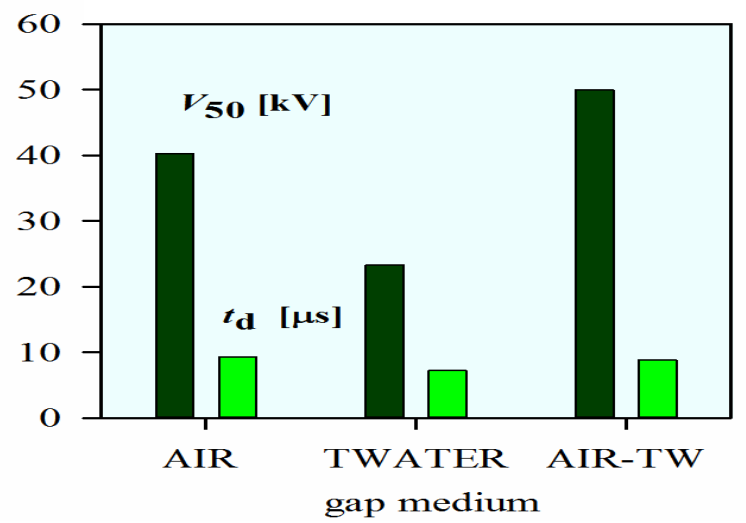

(a) positive rod discharge

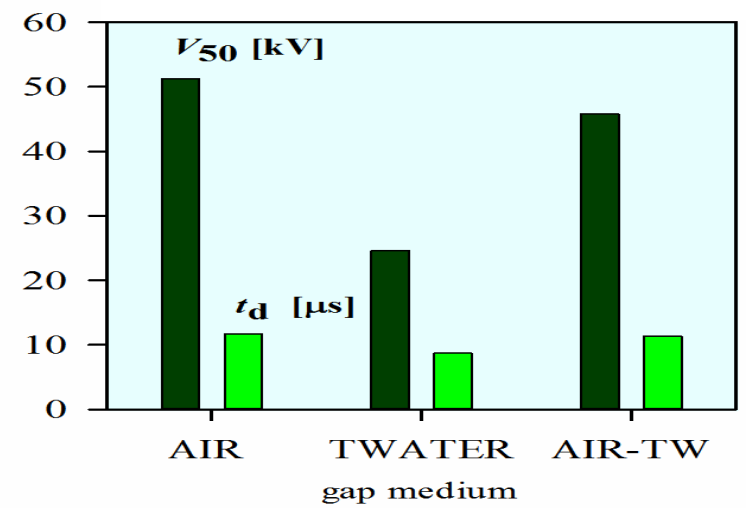

(b) negative rod discharge

Figure 4. Discharge threshold voltage $\left(V_{50}\right)$ and time lag $\left(t_{\mathrm{d}}\right)$ for (a) positive and (b) negative rod discharges

The conditioned discharge waveforms of the voltage $\left(V_{\mathrm{d}}\right)$ and current $\left(I_{\mathrm{d}}\right)$ are shown in Figures 5 and 6 for positive and negative discharges, respectively. These waveforms were determined with the synchronous averaging method using six raw voltage and current waveforms. As a result, smoothed discharge waveforms appeared, and the voltage waveforms indicated different amplitude distortions in both the positive and negative discharges compared to those of the current waveforms. It was confirmed that the period $(T)$ of the voltage and current waveforms was approximately equal to $40 \mu \mathrm{s}$ (corresponding frequency of $25 \mathrm{kHz}$ ). It was also concluded that the value $T$ in the liquid gap was $3 \mu$ s shorter than the case of the airliquid gap reported in [18] and the duration was $57 \%$ of that in the air gap.

The voltage waveform without distortion can be reproduced using the impedance information including in the transient current when the discharge current is replaced by the transient current in the SLIG circuit as shown in eq.(1).

$$
I_{t}(t)=\frac{V_{c}}{L \beta} \exp (-\alpha t) \sin (\beta t)
$$

Where $V_{\mathrm{c}}$ is a charged voltage of $C, \quad \alpha=R_{0} / 2 \mathrm{~L}, \quad$ is total output resistance of the SLIG, $R_{0}<$ $2(L / C)^{0.5}$, and $\beta=\left[1 / L C-\left(R_{0} / 2 L\right)^{2}\right]^{0.5}$. 

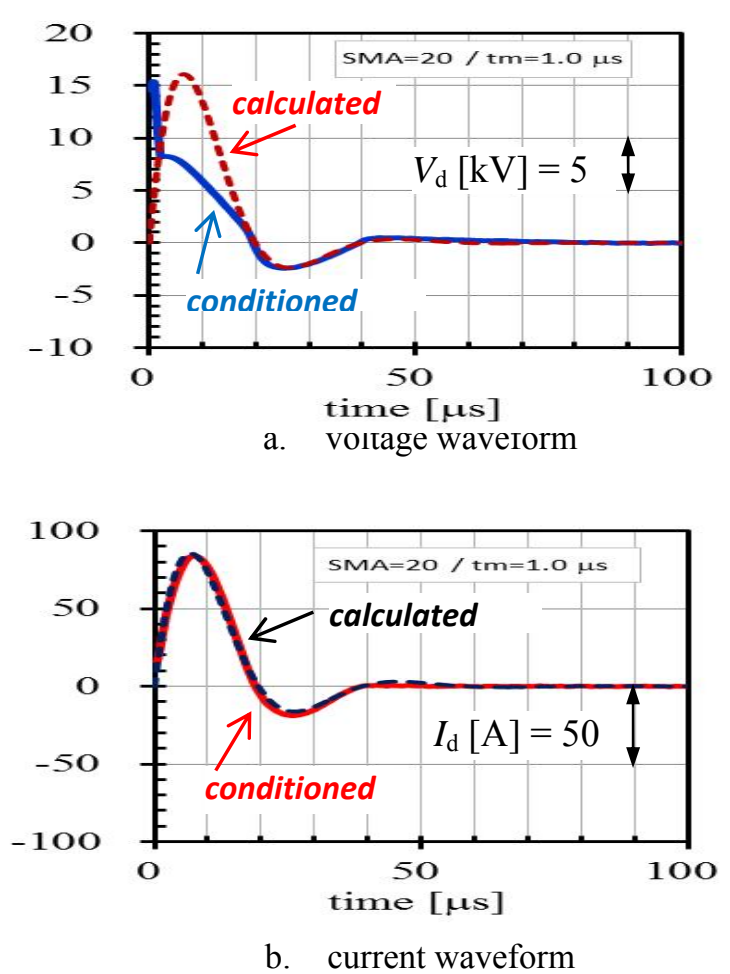

Figure 5. Waveforms for positive rod discharge

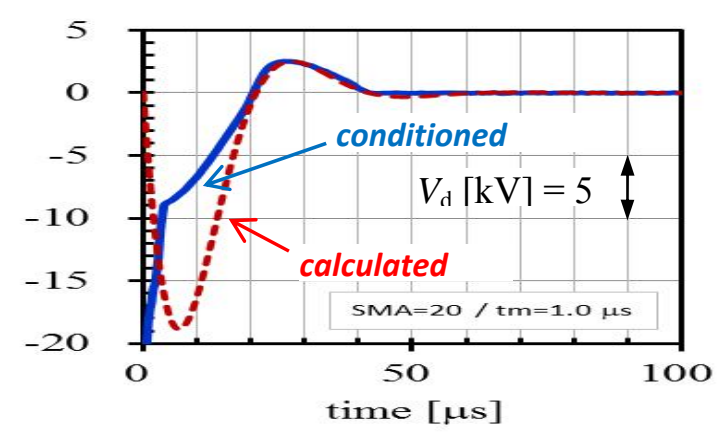

(a) voltagewaveform

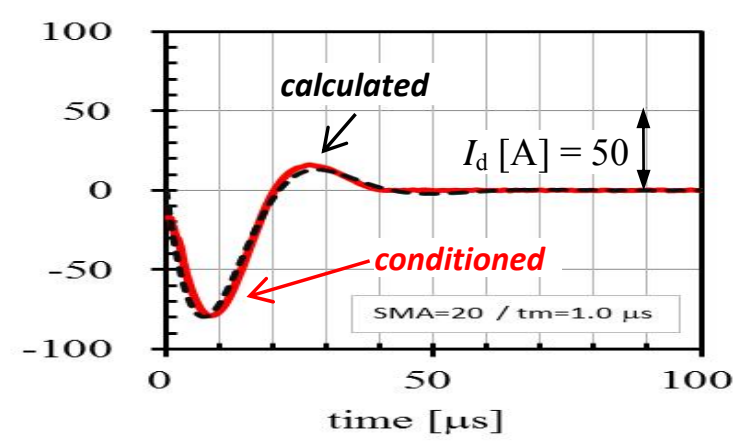

(b) currentwaveform

Figure 6. Waveforms for negative rod discharge 
With respect to the circuit model, a series circuit including the discharge resistance and inductance can be proposed through the experimental results. The existence of the resistance was detected from the oscillating and attenuating waveforms, as shown in Figures 5 and 6 mentioned above. When the $t_{\mathrm{d}}$ was carefully examined, the time lag of all peaks is shown in Figures 7 (a) and (b) for positive and negative discharges, respectively, where air and air-tapwater gaps are also arranged for reference. It was confirmed that the time lag for each current peak in all gaps existed. It was found that the first current peaks each in the positive and negative discharges commonly had a delay time of around $3 \mu$ s against the voltage waveform. Thus, the discharge currents may effectively include an inductive component.

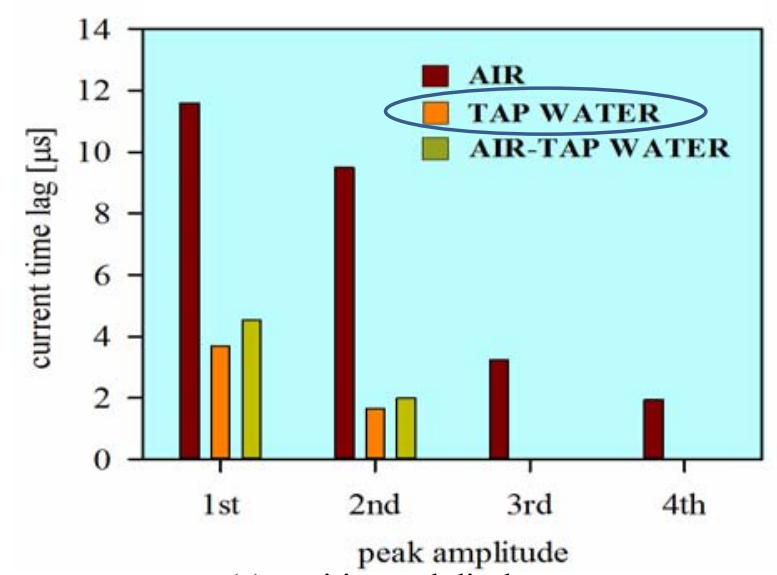

(a) positive rod discharge

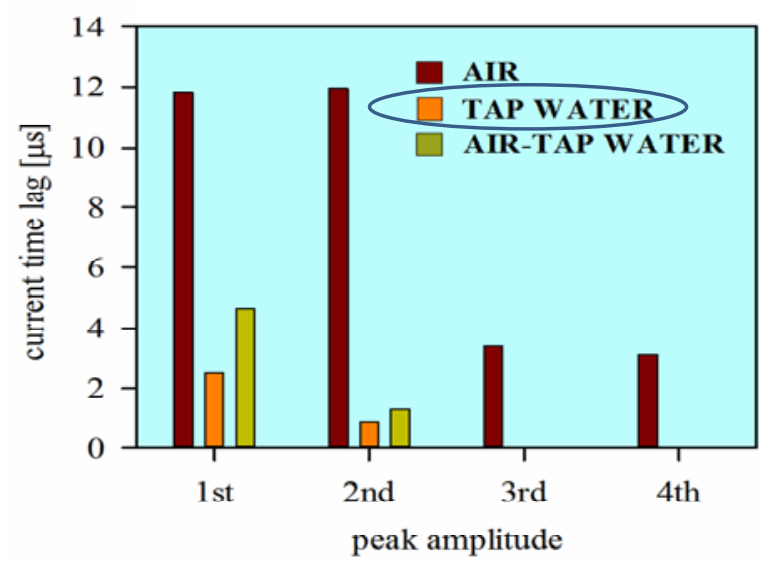

(b) negative rod discharge

Figure 7. Time lag of current to voltage for (a) positive and (b) negative rod discharges in air, tap-water and air-tap-water gaps

The impedance in the tap-water gap is considered for the values obtained by three approaches. The first approach is to calculate the static volume resistance using the liquid conductivity measured in the tap-water gap. The second one is to solve the simultaneous equation with time using the reproduced waveforms, and final one is also to solve the 
simultaneous equation using the conditioned waveforms. The calculation results are shown in Figures 8 (a) and (b) as a function of time for the positive and negative discharges, respectively. The resistance and inductance are represented by $R_{\mathrm{w}}$ and $L_{\mathrm{w}}$, respectively, and the upper solid line is a regression of $L_{\mathrm{w}}$.

The $R_{\mathrm{w}}$ for the first approachwas obtained to be $80 \Omega$ using the conductivity meter, which means the static volume resistanceand is indicated with an arrow. The $R_{\mathrm{w}}$ for the second approach is indicated with a green lower solid curve, and gradually decreased and approached to $80 \Omega$ over time. The $R_{\mathrm{w}}$ for the final approach gradually increased over time but displayed curved regions. The $R_{\mathrm{w}}$ in curved region till $40 \mu$ s partially agreed to the values of the second approach. The $L_{\mathrm{w}}$ value, however, largely oscillated before synchronizing with the current period different from the constant values in the air gap [18]. For times up to $60 \mu$ s, the circuit elements and their impedance can be summarized using the results of the second approach as $R_{\mathrm{w}}=80-200 \Omega, L_{\mathrm{w}}=0.01-31 \mu \mathrm{H}$, and $Z_{\mathrm{w}}=80-200 \Omega$ for the positive discharge; and $R_{\mathrm{w}}=145-$ $255 \Omega, L_{\mathrm{w}}=0.01-140 \Omega \mathrm{H}$, and $Z_{\mathrm{w}}=146-256 \Omega$ for the negative discharge. Then, the value of $R_{\mathrm{w}}$ can be practically exchanged for the value of $Z_{\mathrm{w}}$ even in the liquid gap for the positive and negative rod discharges as well as the case of the low impedance soil in Ref. [14].
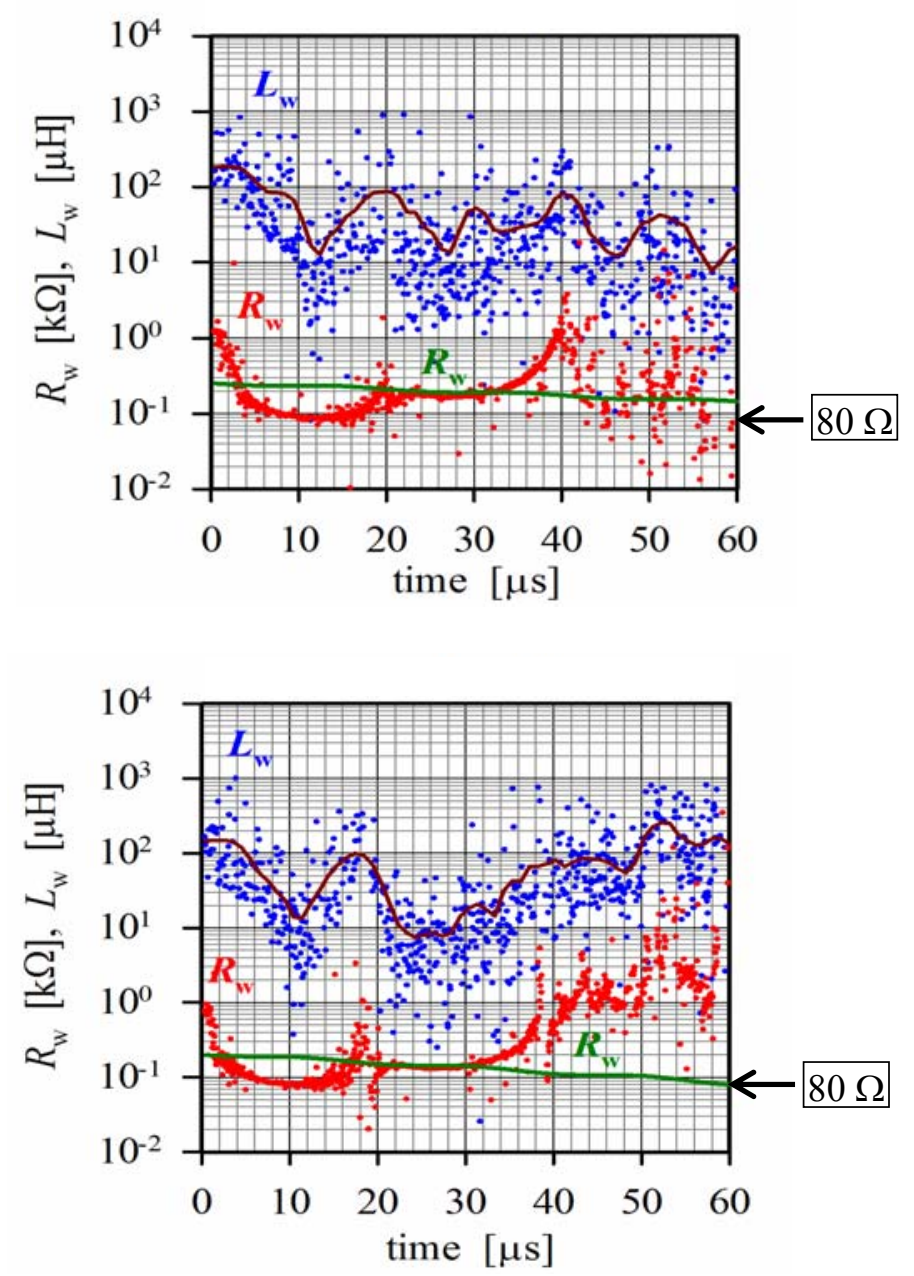

Figure 8. Time dependence of analyzed circuit elements for (a) positive and (b) negative rod discharges in tap-water gap 


\section{Conclusion}

The behaviour of lightning impulse discharge in a liquid gap consisting of tap-water is summarized as follows; (1) the voltage waveform distorted for the first half period; (2) the discharge resistance of fitting curves decreased moderately over time and approached to the static volume resistance for the duration; (3) the discharge impedance was dominated over time by the resistance component because of little inductance one. This may give clues about how to effectively apply an SPD system for internal lightning protection measures.

\section{Acknowledgement}

This work was supported by KAKENHI (no. 23560339) from the Japan Society for the Promotion of Science in the Ministry of Education, Culture, Sports, Science and Technology (MEXT).

\section{References}

[1] https://news.slac.stanford.edu/features/power-generation-blowing-wind (river side)

[2] http://news.xinhuanet.com/english/photo/2013-04/07/c_132290286_2.htm (lake side)

[3] http://www.treehugger.com/renewable-energy/new-report-offshore-wind-could-powerentire-us.html (coast)

[4] http://webberenergyblog.wordpress.com/2010/02/13/offshore-wind-energy-cape-wind-vsvisual-impacts/ (off shore)

[5] B.C. Ummels, M. Gibescu, E. Pelgrum, W.L. Kling, "System Integration of Large-Scale Wind Powerin the Netherlands", in IEEE Power Engineering Society General Meeting, Montreal, Que, 2006, pp.1-8.

[6] G.P. van den Berg, "The Beat is Getting Stronger: The Effect of Atmospheric Stability on Low Frequency Modulated Sound of Wind Turbines", Journal of Low Frequency Noise, Vibration and Active Control, vol. 24, no. 1, pp.1-24, 2005.

[7] V. A. Rakov and M. A. Uman, "Review and Evaluation of Lightning Return Stroke Models Including Some Aspects of Their Application", IEEE Transactions on Electromagnetic Compatibility, vol. 40, pp. 403-426, 1998.

[8] V. A. Rakov, "Some inferences on the propagation mechanisms of dart leaders and return strokes" Journal of Geophysical Research, vol. 103, pp. 1879-1887, 1998.

[9] K. Nakamura, H. Sakurano, Y. Kubouchi, T. Watanabe," Statistical Analysis of Winter Lightning Current and Measurement of Step Voltage in a Wind Power Generation Site", in Proc.31th Int. conf. Lightning Protection, Vienna, Austria, 2012, pp. 1-5.

[10] K.Yamamoto and S.Yanagawa, "Transient Grounding Characteristics of Wind Turbines", in Proc.31th Int. conf. Lightning Protection, Vienna, Austria, 2012, pp. 1-5.

[11] A.N. Hansen, H.V. Erichsen, H.V. Erichsen, "Lightning Protection of the Electrical Systems of WTG: Evaluation Considerations Related to the Application of SPDs", in Proc.31th Int. conf. Lightning Protection, Vienna, Austria, 2012, pp. 1-6.

[12] A. Rousseau, X. Zang, L. Zhang, and M. Tao, "Multiple shots on SPDs - Test Methods and Results", in Proc.31th Int. conf. Lightning Protection, Vienna, Austria, 2012, pp. 1-4.

[13] C. Bouquegneau and B. Jacquet, "How to improve the lightning protection by reducing the ground impedances", in Proc. 17th Int. Conf. on Lightning Protection, Haag, Netherlands, 1983, no.2-3.

[14] R. Alipio and S. Visacro, "How the Frequency Dependence of Soil Parameters Affects the Lightning Response of Grounding Electrodes", in Proc.31th Int. conf. Lightning Protection, Vienna, Austria, 2012, pp. 1-7.

[15] L. Liangfu and Q. Binquan, " Research on Influence of Soil Water Content on Soil Resistivity", in Proc.31th Int. conf. Lightning Protection, Vienna, Austria, 2012, pp. 1-7.

[16] D. Okano, "Simple method of determining plasma impedance of streamer discharge in atmospheric air", Review of Scientific Instruments, vol. 82, pp. 123502(1)-(5), 2011. 
[17] http://www.hach.com/sension5-conductivity-meter-with-electrode-1-meter-cable$230 \mathrm{v} /$ product? $\mathrm{id}=7640486657$ \& callback $=\mathrm{qs}$

[18] D. Okano, "Impedance Property of Lightning Impulse Discharge in Short Composite Gap Consisting of Air and Water", in Proc. 31th Int. Conf. on Lightning Protection, Vienna, Austria, 2012, pp. 1-5.

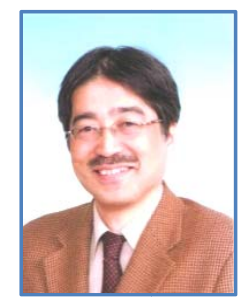

Daisuke Okano received the Ph.D degree in laser diagnostics of plasma from Kyushu University, Japan, in 1984. He is currently a professor of the Liberal Arts Education Center, Tokai University, Kumamoto, Japan. In 1999, he was a Visiting Research Scholar at the University of Florida, FL, USA. His current research interests include the fields of lightning attachment, electromagnetic field analysis by the FDTD method, and lightning protection. 